\title{
Current Concepts and Recent Trends in Arthroscopic Treatment of Large to Massive Rotator Cuff Tears: A Review
}

\author{
Joseph Y. Rho, Yong suk Kwon, Sungwook Choi ${ }^{\circledR}$ \\ Department of Orthopedic Surgery, Jeju National University School of Medicine, Jeju, Korea
}

\begin{abstract}
Rotator cuff tear is a common cause of shoulder pain and disability in adults. Due to the various nature of progression of rotator cuff tears and the complex biomechanics of the shoulder joint, repair and treatment of large-to-massive tears are challenging for many surgeons. Despite the recent popularity of reverse total shoulder arthroplasty as a treatment option for large-to-massive irreparable rotator cuff tears, biological and mechanical repair augmentation has also shown promise as a viable treatment option. The purpose of this study was to briefly summarize and review current studies on the assessment and arthroscopic treatment of large-to-massive rotator cuff tears, whether repairable or irreparable, to aid in developing a consensus on future treatment directions.
\end{abstract}

(Clin Shoulder Elbow 2019;22(1):50-57)

Key Words: Rotator cuff tears; Shoulder; Arthroscopy

\section{Introduction}

Rotator cuff tear is one of the most common causes of shoulder pain and dysfunction. The numbers of outpatient visits and surgeries attributed to symptomatic rotator cuff tears are increasing and both are expected to continue to rise along with the increase in aging of the human population. ${ }^{1-4)}$ Thus far, the overall percentage of rotator cuff tears present in elderly populations ranges from a reported low of $25.6 \%$ to a high of $50.0 \%$. ${ }^{1)}$ One study reported that large-to-massive rotator cuff tears comprise up to $40 \%$ of all rotator cuff tears. ${ }^{3)}$ Arthroscopic repair as a treatment for rotator cuff tears is a popular option and has been widely applied over the past two decades. However, while most small-to-medium tears can be successfully repaired, results of arthroscopic repair of large-to-massive tears are far less favorable. $^{5-10)}$

Numerous surgical treatment options are available for repairing massive rotator cuff tears, including arthroscopic debridement with biceps tenotomy or tenodesis, complete repair, partial repair, patch augmentation, superior capsular reconstruction, muscle tendon transfer, and reverse total shoulder arthroplasty (RTSA). However, there is a lack of agreement on the appropriate treatment guidelines and indications for specific surgical options. $^{3)}$

We acknowledge the complexity of large-to-massive rotator cuff tear treatments due to the numerous considerations and various options available and the difficulties associated with resolving chronic pain and debilitation issues in patients. The purpose of the following is to summarize and review current studies on the assessment and arthroscopic treatment of largeto-massive, reparable and irreparable, rotator cuff tears as an aid to the development of consensus on treatment indications and options.

\section{Patient Selection for Surgical Repair}

Based on the aforementioned complexity and varied nature of large-to-massive rotator cuff tears, evaluating appropriate candidates for operative treatment is crucial for obtaining successful clinical outcomes. ${ }^{11)}$ Unfortunately, even in the presence

Received October 22, 2018. Revised November 21, 2018. Accepted November 27, 2018.

Correspondence to: Sungwook Choi

Department of Orthopedic Surgery, Jeju National University Hospital, 15 Aran 13-gil, Jeju 63241, Korea

Tel: +82-64-754-8116, Fax: +82-64-717-1656, E-mail: swchoi1115@gmail.com, ORCID: https://orcid.org/0000-0003-0319-6208

Review article does not need an IRB approval.

Financial support: None. Conflict of interests: None. 
of quantitative clinical and imaging information, an evidencebased, universally acceptable treatment algorithm is yet to be established. Any proposed clinical evidence-scoring system and treatment algorithm would include both personal experience and scientific data. The following criteria have proven helpful in the assessment of the key parameters in the decision-making process for massive rotator cuff tears in our experience and are offered for consideration. ${ }^{3,11-14)}$

First, the source and nature of pain should be assessed, as well as whether there is any decrease in function. Symptomatic rotator cuff tear patients' most common complaint is pain with overhead activities and pain at night. Initially, it must be determined that the pain is the result of the tear and can be differentiated from concomitant etiologies such as stiffness from adhesive capsulitis and acromioclavicular pain. ${ }^{11)}$ Pain is often associated with weakness and may indicate a large rotator cuff tear; however, weakness is better corroborated through rotator cuff test results. Weakness can be quantified by various semi-objective means and can range from hardly perceived weakness to socalled pseudoparalysis of elevation and/or external rotation., ${ }^{5,11)}$ Recovery from such disability has to be assessed after treatment, and successful reversal of pseudoparalysis through surgery has been reported. ${ }^{15)}$

Second is deciding whether to proceed with treatment or not. Once the source of the pain and/or weakness is determined to be related to rotator cuff failure, and after glenohumeral stiffness and acromioclavicular pain have been excluded, it is ultimately the patient who determines whether the pain and/or disability levels are acceptable to him or her or whether he or she desires improvement. ${ }^{5}$ If the cuff tear is sufficiently disabling, and the patient is willing to undergo operative treatment, it is logical to proceed with treatment because successful repair will provide lasting pain relief and improve strength over that in the preoperative state. ${ }^{16-18)}$

Various classifications of rotator cuff tears have been proposed. However, no consensus currently exists as to which classification system is best, and it is important to interpret the tear pattern information in light of the patient's clinical situation.

Many authors, including Gerber et al., ${ }^{11,17)}$ currently define a cuff tear as massive if there is complete detachment of at least two tendons. Several reports have indicated a higher rate of recurrent tearing for massive rotator cuff tears after surgery compared with that for smaller tears. ${ }^{7,16,19,20)}$ Adding to the complexity of treatment for massive rotator cuff tears is the observation that structural failure does not always equate to clinical failure. In massive cuff tears, if fatty infiltration of the respective muscles is beyond Goutallier stage 2 and/or if there is cranial migration of the humerus resulting in an acromiohumeral distance of less than $7 \mathrm{~mm}$, then the probability that successful cuff repair can be achieved is very low and such massive tears are deemed irreparable. $^{11)}$
To improve the success rates of operative results and thereby clinical outcomes, there are several factors to be considered. ${ }^{21)}$ Azar et al., ${ }^{21)}$ proposed that the following certain aspects are met in order to achieve maximum clinical results. The five aspects are: 1) adequate subacromial decompression, 2) maintaining the integrity of the deltoid origin, 3) mobilizing torn tendons and performing an interval slide, 4) repairing tendons to bone, 5) careful supervision and staging of postoperative rehabilitation. In addition to the factors suggested by Azar et al., ${ }^{21)}$ Yoon $^{4)}$ proposed the following two essential requirements: 1 ) the rotator cuff complex must be able to endure early tension during the early reparative stage (the time between the repair of mechanical properties and tendon formation) and 2) biological ability that maximizes the healing capacity of the bone-tendon junction must be obtained.

In order to achieve such clinical outcome goals, surgical treatment options must provide adequate decompression and debridement of the subacromial space and enough strength in the repaired rotator cuff complex to stabilize the glenohumeral joint, as well as allowing for mobilization and ensuring maximum biological healing capacity of the tear site. ${ }^{21)}$

\section{Surgical Treatment Options}

Developments and advancement in arthroscopic surgery technology have led to the refinement and expansion of surgical indications; thus, massive rotator cuff tears previously considered irreparable can now be considered reparable. Such technological changes have resulted in substantial changes in the approaches to arthroscopic repair of massive rotator cuff tears. ${ }^{14)}$ The following reviews the current treatment options.

\section{Debridement and Biceps Tenotomy}

Traditionally, treatment options for massive rotator cuff tears in elderly patients with low functional demands were limited to arthroscopic debridement. ${ }^{3,11)}$ Although early results were clinically beneficial in alleviating symptoms and improving shoulder range of motion, debridement has become less popular as arthroscopic repair techniques improved and Berth et al. ${ }^{22)}$ studies have determined that debridement produces only temporary benefits. In addition, shoulder strength was likely to remain unchanged or to decrease after debridement. ${ }^{23)}$

The long head of the biceps tendon (LHBT) can be a significant source of shoulder pain, and biceps lesions are often present with massive rotator cuff tears. Greenspoon et al. ${ }^{3)}$ have confirmed that biceps tenotomy or tenodesis can effectively reduce shoulder pain and dysfunction and can produce high satisfaction rates in patients; however, such surgeries do not alter the natural progression of rotator cuff tears. One comparative study showed no significant differences between a group treated with arthroscopic debridement alone and one treated with debride- 
ment plus LHBT tenotomy.

\section{Rotator Cuff Repair}

The aforementioned debridement procedures are usually considered as 'salvage procedures' or 'limited goals surgery'. Although studies have shown debridement can produce satisfactory results in pain relief and range of motion in the elderly, arthroscopic repair should be considered for relatively young or more active patients who have higher functional demand (Fig. 1).

Ideally, a rotator cuff repair would restore cuff biomechanics, decrease pain, improve function, and achieve a strong fixation that would allow an aggressive rehabilitation process and promote healing. While complete anatomic repair, when possible, is the goal, large-to-massive rotator cuff tears remain a clinical challenge due to failure caused by retears. Postoperative recurrent tears of the rotator cuff occur in as little as $11 \%$ of rotator cuff tear study populations but can be as high as 94\%. ${ }^{24)}$ Factors that have been shown to contribute to repair difficulty and retears are increased fatty infiltration of tissue, decreased acromiohumeral distance, smoking, rotator cuff tear size, and increased tension on the repair. ${ }^{3,17,25,26)}$

Accurate recognition of the rotator cuff tear pattern is one of the most critical factors in achieving anatomic reduction and promoting healing, as such knowledge can prevent extreme tensile load at the repair site. ${ }^{14)}$ Also, restoration of balance in the rotator cuff force-couple is another important factor in achieving successful arthroscopic repair of a rotator cuff tear. Disruption of the rotator cuff force-couple may lead to impairment in joint kinetics, ultimately resulting in cartilage and adjacent tendon degeneration. Capsular ligament releases and oblique convergence sutures during arthroscopy are key factors in a satisfactory massive rotator cuff repair and successful clinical outcomes. ${ }^{27-29)}$

The presence of high failure rates of rotator cuff repairs performed via traditional approaches has led to research into and
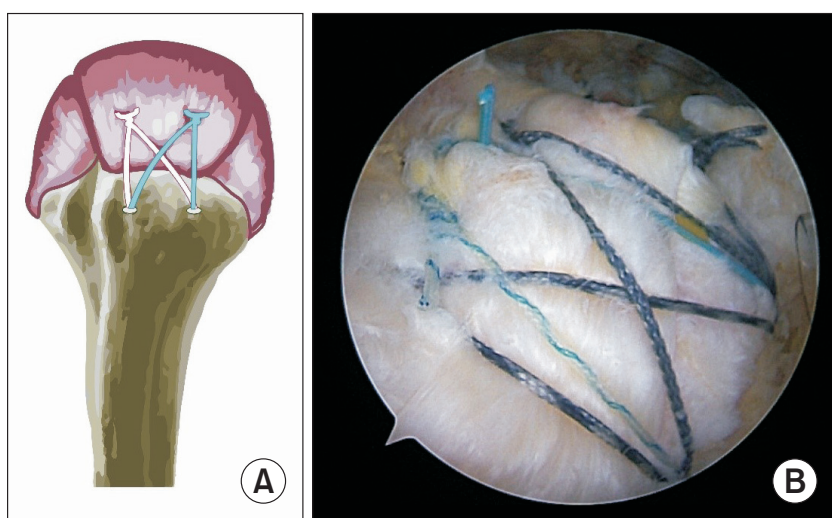

Fig. 1. (A) Schematic illustration of arthroscopic rotator cuff repair with double row suture bridge technique. (B) Repaired rotator cuff via double row suture bridge technique with full coverage of foot print. development of strategies to augment the rotator cuff repair site. The goals of using augmentation in rotator cuff repairs are to enhance mechanical strength through the use of graft tissue and to promote the healing response via biologic augmentation. $^{3,4,9,11,13,14,19,24,30,31)}$

\section{Mechanical and Biological Augmentation of Rotator Cuff Repair}

Various patch augmentation devices have been developed and their use depends on the source or type of graft material selected such as allograft, xenograft, and synthetic extracellular matrix (ECM) scaffold grafts. ${ }^{13)}$ Although clear indications for the use of scaffold devices in rotator cuff repair have not been defined, augmentation through the use of ECM patches is being widely investigated as a treatment option for large-to-massive rotator cuff tears.

Allografts and nonhuman mammalian ECM scaffold devices have potential mechanical and biologic benefits in rotator cuff repair. Scaffold augmentation can provide adequate mechanical integrity during the early phase of rotator cuff repair healing and can serve as a base for cell migration and enhanced collagenous matrix production, which can ensure tendon-to-bone healing at the repair site. Synthetic scaffolds may not provide a direct biologic effect during healing; however, they are able to maintain durable mechanical properties over time and stabilize the repair construct until tissue healing occurs. ${ }^{11,14,32,33)}$

Authors of prospective studies of human dermis-derived allograft scaffolds have reported improved clinical scores and significantly higher rotator cuff integrity in their augmentation group that in their nonaugmentation group (85\% vs. $40 \%) .{ }^{34-36)} \mathrm{A}$ histologic assessment of an allogenically augmented rotator cuff repair site demonstrated no calcification, infection, or inflammatory response at 3 months after repair. However, this is not to say that allogenic ECM augmentation is without potential concern as allogenic ECMs can produce inflammatory responses in the host. Moreover, they are less elastic than autogenic tendons, which may result in a comparably higher retear rate due to the lower load-carrying ability of the ECM. ${ }^{14,34)}$

Immunogenicity is of great concern when using ECM-based xenografts derived from a nonhuman species. ${ }^{14)}$ Despite precautions and adequate graft preparation procedures involving rigorous decellularization, studies using xenograft ECM made from porcine small intestine mucosa have reported a high proportion of patients exhibiting severe inflammatory reactions and have recommended against the use of such augmentation. ${ }^{37,38)}$ On the other hand, Throckmorton and Gerlinger ${ }^{13)}$ have reported favorable outcomes with dermal-origin xenografts. Throckmorton and Gerlinger ${ }^{13)}$ studies have also reported significant improvements in clinical outcome scores, range of motion, and strength.

Due to concerns associated with xenografts and the associated risk of immunological responses, synthetic polymer materi- 
als have emerged as a possible graft option. The consistency and strength of synthetic ECMs indicate they are potential candidate augmentation materials that can allow cellular and fibrotic growth of rotator cuff repairs. Studies have shown significantly improved function and intact rotator cuff repairs that were augmented with synthetic ECM grafts. ${ }^{13,39)}$ One study reported retearing rates at follow-up 1 year after repair of $17 \%, 51 \%$, and $41 \%$ in patients with synthetic graft augmentation, xenograft augmentation, and no augmentation, respectively. ${ }^{14,40)}$

Mechanical repair and enhancement are important mainstays in rotator cuff tear repair. Although functional outcomes in patients who undergo rotator cuff repair may remain relatively good and despite ultrasonographic or magnetic resonance imaging evidence of recurrent tears, the presence of a correlation between healing at the repair site and improved functional outcome cannot be neglected. Russell et al. ${ }^{41)}$ reported on patients with an intact rotator cuff repair and patients with a retear after repair; after a minimum follow-up of 1 year, the patients with an intact repair had better mean Constant-Murley and University of California at Los Angeles shoulder rating scores.

Elucidation of the biology of healing of the rotator cuff and cellular healing at patch-augmented repair sites has led to research considering more direct therapies (Fig. 2). Numerous animal studies investigating the application of cellular growth factors during healing of rotator cuff repair sites have reported increased cell proliferation, neovascularization, and bone volume. However, these studies have yet to show observable, significant improvement in the mechanical quality of the repair or an increase in tendon load prior to failure. Cheung et al. ${ }^{24)}$ indicated that, in vivo, growth factors may function via interaction with other factors to ultimately enhance tendon-to-bone healing, and that it is unlikely a significant change will be observable after applying only one growth factor.

Platelet-rich plasma (PRP) is an autologous whole bloodderived concentrate that contains multiple growth factors, many of them previously identified as crucial in normal bone-totendon healing. ${ }^{42)}$ The growth factors in PRP include plateletderived growth factor, transforming growth factor- $\beta$, vascular endothelial growth factor, and insulin-like growth factor- $1 .{ }^{43)}$ The administration of PRP at the rotator cuff repair site may promote healing via such factors; however, there is controversy over the role of PRP as a biologic augmentation source due to the reporting of conflicting results on PRP effects on tendon healing. ${ }^{14)}$ Recent systematic reviews and meta-analyses have reported no significant difference in clinical outcome after PRP treatment, thus there is a lack of evidence indicating a benefit of PRP augmentation in rotator cuff repair. ${ }^{44-47}$ Numerous questions on the optimal timing, dosing, mode of application, and formulation of PRP treatment in rotator cuff repair need to be investigated, and the results of such studies may help define the clinical benefit and indications for the use of PRP. ${ }^{14)}$

\section{Superior Capsular Reconstruction}

Patches have been used successfully to augment primary repairs, but they are insufficient in providing the structural integrity needed to span the gap within the setting of an irreparable tear. ${ }^{3)}$ Partial repair is another option, but the risk of retear can be as high as $52 \% .{ }^{7,47)}$ RTSA has gained popularity as it is effective in
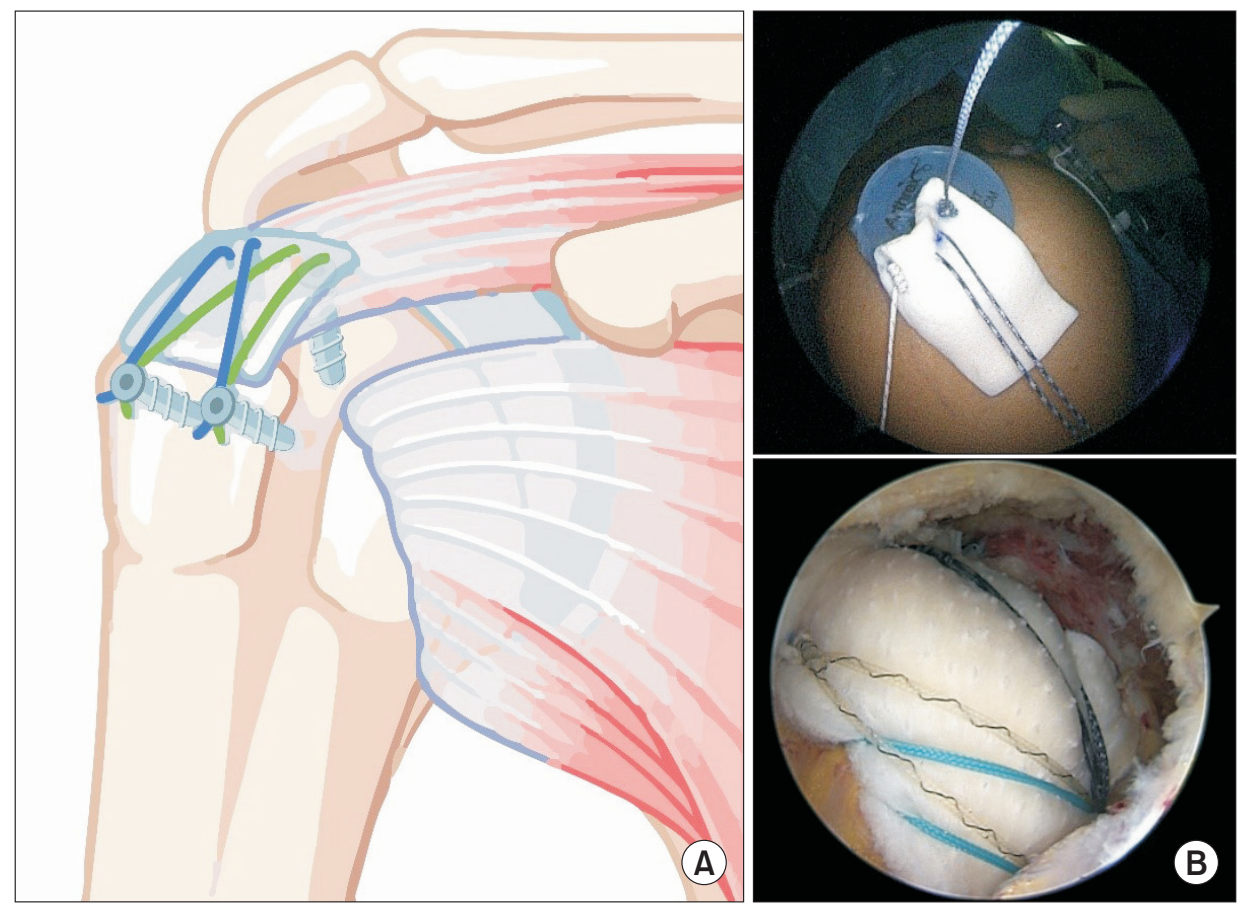

Fig. 2. (A) Schematic illustration of rotator cuff repair with allogenous dermal patch graft augmentation. (B) Repaired rotator cuff via single row suture technique with allogenous dermal patch graft augmentation. 

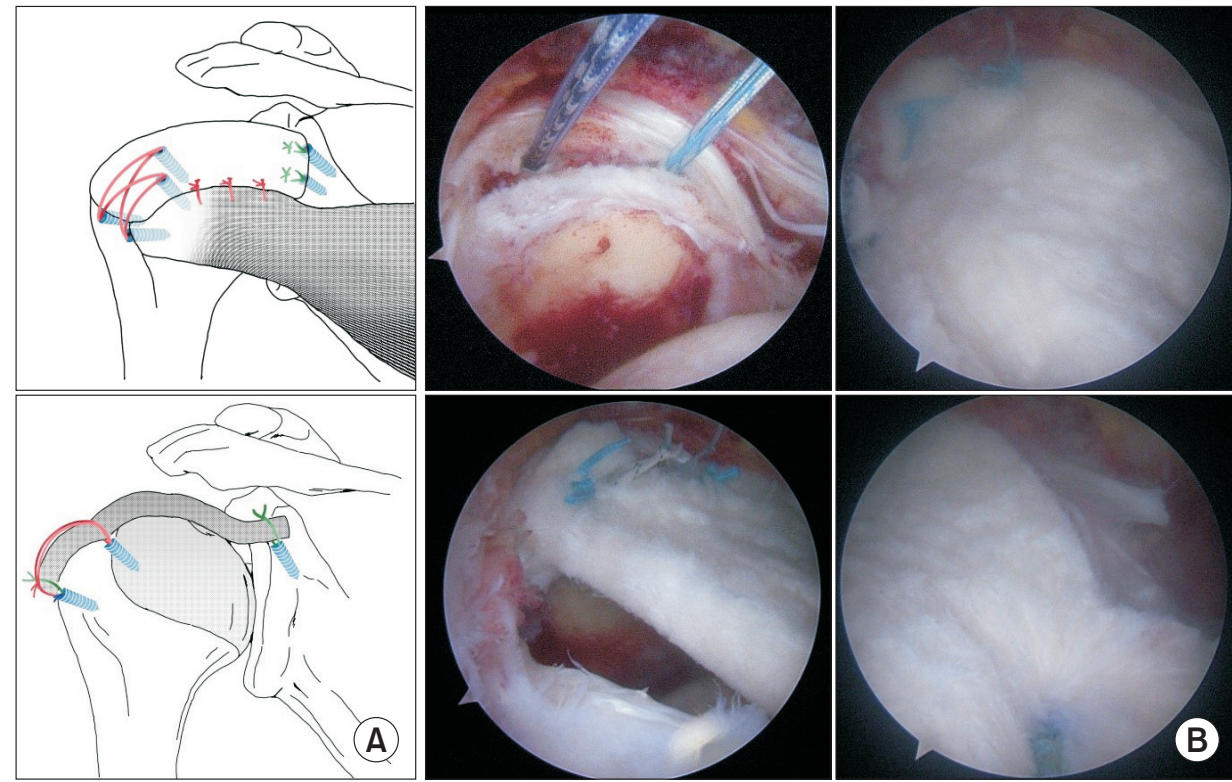

Fig. 3. (A) Schematic illustration of superior capsular reconstruction in irreparable massive rotator cuff tear. (B) Irreparable rotator cuff defect is filled by arthroscopic superior capsular reconstruction using allogenous dermal patch graft. the treatment of rotator cuff tears in elderly patients; however, it has failed to provide similar clinical results in younger patients. $^{14,48,49)}$

Superior capsule reconstruction (SCR) is an option that has the potential to restore and rebalance the force couples necessary for dynamic shoulder function (Fig. 3). ${ }^{50)}$ Recently, biomechanical studies have shown that the superior capsule is a critical static stabilizer that enables the glenohumeral joint to be reduced, allowing larger muscles like the deltoid and pectoralis major to function properly. ${ }^{50-52)}$ Reconstruction of the superior capsule with a collagen graft, attached medially to the superior glenoid and laterally to the greater tuberosity, was shown to restore the superior capsule to physiological conditions in a cadaveric study. ${ }^{52)}$ This reconstruction method can provide a treatment option suitable for patients with an irreparable rotator cuff tear; in addition, it does not compromise future treatment options.

In order to restore the gap and cavitary defect in the superior capsule and cuff area, Mihata et al. ${ }^{53)}$ initially suggested an SCR technique that used a folded fascia lata graft. Their original technique was distinct from previous methods because, medially, the graft was secured to the superior glenoid neck (forming a graft-to-bone interface) rather than bridging the gap between the remnant rotator cuff and its lateral insertion on the greater tuberosity (forming a graft-to-tendon interface). The early results of Mihata et al. ${ }^{53)}$ were promising, but the fascia lata graft is relatively thin and a large donor incision is needed for harvesting; as a consequence, alternative SCR methods have been investigated. ${ }^{6,14,28,50)}$

Several authors have described wholly arthroscopic SCR techniques using acellular dermal allografts. ${ }^{6,27,50,51)}$ Such a method has advantages over that using a fascia lata autograft. By using acellular dermal allografts, donor harvest site morbid- ity is avoided and biologic incorporation can be achieved while maintaining structural integrity. ${ }^{14)}$ The surgical steps in SCR are fairly universal although, depending on the study authors, there are variations and differences in suggested improvements.

Early preliminary results for allograft reconstruction of superior capsules are encouraging. Burkhart et al. ${ }^{28)}$ reported clinical outcome improvements in American Shoulder and Elbow Surgeons and visual analogue scale scores from 45.6 to 85.8 and 5.26 to 0.96 , respectively, at one year after surgery. One of the disadvantages of SCR performed using an allodermal patch is that it is a technically demanding procedure. Recently, a technical modification of SCR that used an LHBT autograft for patients whose LHBT integrity is preserved was suggested. ${ }^{54,55)}$ Boutsiadis et al. ${ }^{54)}$ introduced an LHBT transfer technique. In that approach, the tendon's insertion into the glenoid is left intact, while laterally, it is tenotomized, transferred, and sutured with anchors onto the footprint of the supraspinatus tendon, thereby acting as a superior static stabilizer of the shoulder joint. ${ }^{54}$

A study reported by Kim et al. ${ }^{55)}$ described a biceps tendon rerouting technique for large-to-massive rotator cuff tears. In that technique, lasso-loop ties are made through the body of the LHBT and fixed at the footprint of the greater tuberosity. Initially, the LHBT is mobilized and surrounding soft tissue is removed. After a lateral anchor is inserted to affix the LHBT, one lassoloop and two wrap-around ties are made at the lateral anchor. ${ }^{55}$ Another anchor is then inserted and fixed medially with the additional anchor fixed just posterior to the lateral LHBT anchor. ${ }^{55)}$ The LHBT can be tenotomized at the distal aspect of the lateral anchor if tendon integrity is insufficient to maintain itself. An increased acromiohumeral distance indicating downward migration of the humeral head in immediate postoperative radiographs suggested that the LHBT rerouting technique may 
improve static stability for the glenohumeral joint of a shoulder with a large-to-massive rotator cuff tear. ${ }^{14,55)}$ Utilizing the LHBT in SCR has advantages as it can be performed as an 'all-inside' procedure that is technically less demanding, less expensive, and has a reduced risk of infection due to a lack of donor site morbidity. ${ }^{14)}$

\section{Conclusion}

Rotator cuff tears are an increasingly common source of shoulder pain and disability among patients. Although the repair and treatment of a rotator cuff tear is not simple, increased description and elucidation of the biomechanics of the shoulder joint, appreciation of the importance of force-couple balances, and recognition and management of rotator cuff tear patterns has resulted in substantial improvements in the approaches used for arthroscopic repair of massive rotator cuff tears. Despite the popularity of RTSA as a treatment option for large-to-massive irreparable rotator cuff tears, biological and/or mechanical augmentation of repair constructs are considered viable treatment options. In addition, recent research into SCR using either allograft patch or the LHBT has shown promising short-term clinical results. However, further research and verification of the potential of using PRP, growth factors, and stem cell augmentation methods are required.

\section{Acknowledegments}

The schematic illustrations in this study's figures were prepared by Sunjoo Park (Fig. 1, 2) and Jiwon Ha (Fig. 3).

\section{References}

1. Yamamoto A, Takagishi K, Osawa T, et al. Prevalence and risk factors of a rotator cuff tear in the general population. J Shoulder Elbow Surg. 2010;19(1):116-20. doi: 10.1016/j.jse. 2009.04.006.

2. Jeong J, Shin DC, Kim TH, Kim K. Prevalence of asymptomatic rotator cuff tear and their related factors in the Korean population. J Shoulder Elbow Surg. 2017;26(1):30-5. doi: 10.1016/ j.jse.2016.05.003.

3. Greenspoon JA, Petri M, Warth RJ, Millett PJ. Massive rotator cuff tears: pathomechanics, current treatment options, and clinical outcomes. J Shoulder Elbow Surg. 2015;24(9):1493505. doi: 10.1016/j.jse.2015.04.005.

4. Yoon JP. Patch Augmentation for Massive Rotator Cuff Tears. Clin Should Elbow. 2017;20(2):105-12. doi: 10.5397/cise. 2017.20.2.105.

5. Henry P, Wasserstein D, Park S, et al. Arthroscopic repair for chronic massive rotator cuff tears: a systematic review. Arthroscopy. 2015;31(12):2472-80. doi: 10.1016/ j.arthro.2015.06.038.

6. Hirahara AM, Adams CR. Arthroscopic superior capsular reconstruction for treatment of massive irreparable rotator cuff tears. Arthrosc Tech. 2015;4(6):e637-41. doi: 10.1016/ j.eats.2015.07.006.

7. Duralde XA, Bair B. Massive rotator cuff tears: the result of partial rotator cuff repair. J Shoulder Elbow Surg. 2005;14(2):1217. doi: 10.1016/j.jse.2004.06.015.

8. Jones CK, Savoie FH 3rd. Arthroscopic repair of large and massive rotator cuff tears. Arthroscopy. 2003;19(6):564-71. doi: 10.1016/S0749-8063(03)00169-5.

9. MacDonald PB, Altamimi S. Principles of arthroscopic repair of large and massive rotator cuff tears. Instr Course Lect. 2010;59:269-80.

10. Valenti P. Joint-preserving treatment options for irreparable rotator cuff tears. Orthopade. 2018;47(2):103-12. doi: 10.1007/ s00132-017-3516-1.

11. Gerber C, Wirth SH, Farshad M. Treatment options for massive rotator cuff tears. J Shoulder Elbow Surg. 2011;20(2 Suppl):S20-9. doi: 10.1016/j.jse.2010.11.028.

12. Oh LS, Wolf BR, Hall MP, Levy BA, Marx RG. Indications for rotator cuff repair: a systematic review. Clin Orthop Relat Res. 2007;455:52-63. doi: 10.1097/BLO.0b013e31802fc175.

13. Throckmorton TQ, Gerlinger TL. Instructional course lectures. Rosemont (IL): American Academy of Orthopaedic Surgeons; 2016. 93-108.

14. Parvizi J, Huddleston JI. Instructional course lectures. Rosemont (IL): American Academy of Orthopaedic Surgeons; 2018. 129-142.

15. Mihata T, Lee TQ, Hasegawa A, et al. Arthroscopic superior capsule reconstruction can eliminate pseudoparalysis in patients with irreparable rotator cuff tears. Am J Sports Med. 2018;46(11):2707-16. doi: 10.1177/0363546518786489.

16. Bigliani LU, Cordasco FA, McLlveen SJ, Musso ES. Operative repair of massive rotator cuff tears: long-term results. J Shoulder Elbow Surg. 1992;1(3):120-30. doi: 10.1016/10582746(92)90089-L.

17. Gerber C, Fuchs B, Hodler J. The results of repair of massive tears of the rotator cuff. J Bone Joint Surg Am. 2000;82(4):50515.

18. Lam F, Mok D. Open repair of massive rotator cuff tears in patients aged sixty-five years or over: is it worthwhile? J Shoulder Elbow Surg. 2004;13(5):517-21. doi: 10.1016/ j.jse.2004.02.016.

19. Neri BR, Chan KW, Kwon YW. Management of massive and irreparable rotator cuff tears. J Shoulder Elbow Surg. 2009;18(5):808-18. doi: 10.1016/j.jse.2009.03.013.

20. Bedi A, Dines J, Warren RF, Dines DM. Massive tears of the rotator cuff. J Bone Joint Surg Am. 2010;92(9):1894-908. doi: 10.2106/JBJS.I.01531.

21. Azar FM, Canale ST, Beaty JH. Campbell's operative orthopae- 
dics. 13th ed. Amsterdam: Elsevier; 2016. 2298-2345.

22. Berth A, Neumann W, Awiszus F, Pap G. Massive rotator cuff tears: functional outcome after debridement or arthroscopic partial repair. J Orthop Traumatol. 2010;11(1):13-20. doi: 10.1007/s10195-010-0084-0.

23. Burkhart SS. Arthroscopic treatment of massive rotator cuff tears. Clin Orthop Relat Res. 2001;(390):107-18.

24. Cheung EV, Silverio L, Sperling JW. Strategies in biologic augmentation of rotator cuff repair: a review. Clin Orthop Relat Res. 2010;468(6):1476-84. doi: 10.1007/s11999-010-13237.

25. Khatri C, Ahmed I, Parsons H, et al. The natural history of full-thickness rotator cuff tears in randomized controlled trials: a systematic review and meta-analysis. Am J Sports Med. In press, available on 2 July 2018. doi: 10.1177/0363546518780694.

26. Nganga M, Lizarondo L, Krishnan J, Stephenson M. Management of full thickness rotator cuff tears in the elderly: a systematic review protocol. JBI Database System Rev Implement Rep. 2018;16(8):1628-33. doi: 10.11124/JBISRIR-2017-003596.

27. Adams CR, Denard PJ, Brady PC, Hartzler RU, Burkhart SS. The arthroscopic superior capsular reconstruction. Am J Orthop (Belle Mead NJ). 2016;45(5):320-4.

28. Burkhart SS, Denard PJ, Adams CR, Brady PC, Hartzler RU. Arthroscopic superior capsular reconstruction for massive irreparable rotator cuff repair. Arthrosc Tech. 2016;5(6):e140718. doi: 10.1016/j.eats.2016.08.024.

29. Burkhart SS. Arthroscopic repair of massive rotator cuff tears: concept of margin convergence. Tech Shoulder Elb Surg. 2000;1(4):232-9.

30. Cofield RH, Parvizi J, Hoffmeyer PJ, Lanzer WL, Ilstrup DM, Rowland CM. Surgical repair of chronic rotator cuff tears. A prospective long-term study. J Bone Joint Surg Am. 2001;83(1):71-7.

31. Dang A, Davies M. Rotator cuff disease: treatment options and considerations. Sports Med Arthrosc Rev. 2018;26(3):129-33. doi: 10.1097/JSA.0000000000000207.

32. Wong I, Burns J, Snyder S. Arthroscopic GraftJacket repair of rotator cuff tears. J Shoulder Elbow Surg. 2010;19(2 Suppl):104-9. doi: 10.1016/j.jse.2009.12.017.

33. Gilot GJ, Alvarez-Pinzon AM, Barcksdale L, Westerdahl D, Krill M, Peck E. Outcome of large to massive rotator cuff tears repaired with and without extracellular matrix augmentation: a prospective comparative study. Arthroscopy. 2015;31(8):145965. doi: 10.1016/j.arthro.2015.02.032.

34. Barber FA, Burns JP, Deutsch A, Labbé MR, Litchfield RB. A prospective, randomized evaluation of acellular human dermal matrix augmentation for arthroscopic rotator cuff repair. Arthroscopy. 2012;28(1):8-15. doi: 10.1016/ j.arthro.2011.06.038.

35. Denard PJ, Jiwani AZ, Lädermann A, Burkhart SS. Long-term outcome of arthroscopic massive rotator cuff repair: the importance of double-row fixation. Arthroscopy. 2012;28:909-15. doi: 10.1016/j.arthro.2011.12.007.

36. Vaishnav S, Millett PJ. Arthroscopic rotator cuff repair: scientific rationale, surgical technique, and early clinical and functional results of a knotless self-reinforcing double-row rotator cuff repair system. J Shoulder Elbow Surg. 2010;19(2 Suppl):83-90. doi: 10.1016/j.jse.2009.12.012.

37. Walton JR, Bowman NK, Khatib Y, Linklater J, Murrell GA. Restore orthobiologic implant: not recommended for augmentation of rotator cuff repairs. J Bone Joint Surg Am. 2007;89(4):786-91.

38. James KS, Cornwell KC, Greenburg AG. Extracellular matrix bioscaffolds for orthopaedic applications. J Bone Joint Surg Am. 2010;92(5):1316-7; author reply 1317-8.

39. Lederman ES, Toth AP, Nicholson GP, et al. A prospective, multicenter study to evaluate clinical and radiographic outcomes in primary rotator cuff repair reinforced with a xenograft dermal matrix. J Shoulder Elbow Surg. 2016;25(12):1961-70. doi: 10.1016/j.jse.2016.02.029.

40. Ciampi P, Scotti C, Nonis A, et al. The benefit of synthetic versus biological patch augmentation in the repair of posterosuperior massive rotator cuff tears: a 3-year followup study. Am J Sports Med. 2014;42(5):1169-75. doi: 10.1177/0363546514525592.

41. Russell RD, Knight JR, Mulligan E, Khazzam MS. Structural integrity after rotator cuff repair does not correlate with patient function and pain: a meta-analysis. J Bone Joint Surg Am. 2014;96(4):265-71. doi: 10.2106/JBJS.M.00265.

42. Boswell SG, Cole BJ, Sundman EA, Karas V, Fortier LA. Platelet-rich plasma: a milieu of bioactive factors. Arthroscopy. 2012;28(3):429-39. doi: 10.1016/j.arthro.2011.10.018.

43. Kobayashi $M$, Itoi $E$, Minagawa $H$, et al. Expression of growth factors in the early phase of supraspinatus tendon healing in rabbits. J Shoulder Elbow Surg. 2006;15(3):371-7. doi: 10.1016/j.jse.2005.09.003.

44. Jo CH, Shin JS, Lee YG, et al. Platelet-rich plasma for arthroscopic repair of large to massive rotator cuff tears: a randomized, single-blind, parallel-group trial. Am J Sports Med. 2013;41(10):2240-8. doi: 10.1177/0363546513497925.

45. Gumina S, Campagna V, Ferrazza G, et al. Use of plateletleukocyte membrane in arthroscopic repair of large rotator cuff tears: a prospective randomized study. J Bone Joint Surg Am. 2012;94(15):1345-52. doi: 10.2106/JBJS.K.00394.

46. Holtby R, Christakis M, Maman E, et al. Impact of plateletrich plasma on arthroscopic repair of small- to mediumsized rotator cuff tears: a randomized controlled trial. Orthop J Sports Med. 2016;4(9):2325967116665595. doi: $10.1177 / 2325967116665595$.

47. Chahal J, Van Thiel GS, Mall N, et al. The role of platelet-rich plasma in arthroscopic rotator cuff repair: a systematic review 
with quantitative synthesis. Arthroscopy. 2012;28(11):1718-27. doi: 10.1016/j.arthro.2012.03.007.

48. Kim SH, Wise BL, Zhang Y, Szabo RM. Increasing incidence of shoulder arthroplasty in the United States. J Bone Joint Surg Am. 2011;93(24):2249-54. doi: 10.2106/JBJS.J.01994.

49. Schairer WW, Nwachukwu BU, Lyman S, Craig EV, Gulotta LV. National utilization of reverse total shoulder arthroplasty in the United States. J Shoulder Elbow Surg. 2015;24(1):91-7. doi: 10.1016/j.jse.2014.08.026.

50. Petri M, Greenspoon JA, Millett PJ. Arthroscopic superior capsule reconstruction for irreparable rotator cuff tears. Arthrosc Tech. 2015;4(6):e751-5. doi: 10.1016/j.eats.2015.07.018.

51. Tokish JM, Beicker C. Superior capsule reconstruction technique using an acellular dermal allograft. Arthrosc Tech. 2015;4(6):e833-9. doi: 10.1016/j.eats.2015.08.005.

52. Mihata T, McGarry MH, Pirolo JM, Kinoshita M, Lee TQ. Superior capsule reconstruction to restore superior stabil- ity in irreparable rotator cuff tears: a biomechanical cadaveric study. Am J Sports Med. 2012;40(10):2248-55. doi: $10.1177 / 0363546512456195$.

53. Mihata T, Lee TQ, Watanabe $C$, et al. Clinical results of arthroscopic superior capsule reconstruction for irreparable rotator cuff tears. Arthroscopy. 2013;29(3):459-70. doi: 10.1016/ j.arthro.2012.10.022.

54. Boutsiadis A, Chen S, Jiang C, Lenoir H, Delsol P, Barth J. Long head of the biceps as a suitable available local tissue autograft for superior capsular reconstruction: "The Chinese Way". Arthrosc Tech. 2017;6(5):e1559-66. doi: 10.1016/ j.eats.2017.06.030.

55. Kim YS, Lee HJ, Park I, Sung GY, Kim DJ, Kim JH. Arthroscopic in situ superior capsular reconstruction using the long head of the biceps tendon. Arthrosc Tech. 2018;7(2):e97-103. doi: 10.1016/j.eats.2017.08.058. 